\title{
BMJ Global Health Predicting the impact of control strategies on the tuberculosis burden in South and North Korea using a mathematical model
}

\author{
Hyunwoo Cho, ${ }^{1}$ Youngmok Park, ${ }^{2}$ Jeongjoo Seok, ${ }^{1}$ Joon Sup Yeom (D) , ${ }^{3}$ \\ Jun Yong Choi, ${ }^{2}$ Hee Jin Kim, ${ }^{4}$ Young Ae Kang, ${ }^{2,5}$ Jeehyun Lee ${ }^{1}$
}

To cite: Cho H, Park Y,

Seok J, et al. Predicting the impact of control strategies on the tuberculosis burden in South and North Korea using a mathematical model. BMJ Global Health 2021;6:e005953. doi:10.1136/ bmjgh-2021-005953

Handling editor Seye Abimbola

- Additional supplemental material is published online only. To view, please visit the journal online (http://dx.doi.org/10. 1136/bmjgh-2021-005953)

YAK and JL contributed equally.

Received 8 April 2021

Accepted 14 September 2021

Check for updates

C Author(s) (or their employer(s)) 2021. Re-use permitted under CC BY-NC. No commercial re-use. See rights and permissions. Published by BMJ.

For numbered affiliations see end of article.

\section{Correspondence to}

Professor Jeehyun Lee; ezhyun@yonsei.ac.kr and Professor Young Ae Kang; MDKANG@yuhs.ac

\section{ABSTRACT}

Background Among high-income countries, South Korea has a considerable tuberculosis (TB) burden; North Korea has one of the highest TB burdens in the world. Predicting the impact of control strategies on the TB burden can help to efficiently implement TB control programmes.

Methods We designed a deterministic compartmental model of TB in Korea. After calibration with notification of incidence data from South Korea, the TB burden for 2040 was predicted according to four different intervention strategies: latent TB infection (LTBI) treatment, rapid diagnosis, active case-finding and improvement of the treatment success rate. North Korea's burden in 2040 was similarly estimated by adjusting the model parameters.

Results In South Korea, the number of patients with drugsusceptible TB (DS-TB) and multidrug-resistant TB (MDRTB) were predicted to be 27581 and 625 , respectively, in 2025. Active case-finding would lower DS-TB by $6.2 \%$ and MDR-TB by $26.7 \%$, respectively, in 2040 . The improvement in the success rate of DS-TB treatment would reduce the MDR-TB burden by $34.5 \%$. In North Korea, the number of patients with DS-TB and MDR-TB are, respectively, predicted to be 77629 and 5409 in 2025. Active casefinding would reduce DS-TB by $22.2 \%$ and MDR-TB by $69.7 \%$. LTBI treatment would reduce DS-TB by $20.6 \%$ and MDR-TB by $38.6 \%$.

Conclusion The impact of control strategies on the TB burden in South and North Korea was investigated using a mathematical model. The combined intervention strategies would reduce the burden and active case-finding is expected to result in considerable reduction in both South and North Korea.

\section{INTRODUCTION}

Tuberculosis (TB), a communicable disease that is a major cause of ill health, is one of the top 10 causes of death worldwide and the leading cause of death from a single infectious agent. ${ }^{1}$ Despite efforts to eliminate TB based on the WHO's END TB strategy ${ }^{2}$ and a political declaration at a UN high-level meeting, ${ }^{3}$ an estimated 10 million people suffered from TB in 2018. Moreover, drug-resistant

\begin{abstract}
WHAT IS ALREADY KNOWN?
$\Rightarrow$ Tuberculosis(TB) is a significant challenge in the Korean peninsula. North Korea is one of the 30 countries with the highest TB and drug resistant TB burdens and TB incidence in South Korea is considerable among high income countries.

$\Rightarrow$ There are relatively few studies on the modelling of drug resistant TB incorporating to the drug susceptible TB.

\section{WHAT ARE THE NEW FINDINGS?}

$\Rightarrow$ Using reported national TB data from South and North Korea, local epidemiologic parameters, we developed a dynamic model for drug susceptible (DS) and multidrug-resistant (MDR)TB in South and North Korea. DS and MDR-TB continuously decreased and active case finding (ACF) and the improvement of DS-TB treatment were effective in reducing TB burdens in South Korea. In North Korea, MDR-TB burdens increased even several interventions. ACF and improvement of diagnostics and DS-TB treatment were effective in reducing MDR-TB in North Korea.

\section{WHAT DO THE NEW FINDINGS IMPLY?}

$\Rightarrow$ The DS-TB and MDR-TB incorporating model we developed can help estimate the impact of TB control strategies comprehensively. In resource limited settings such as North Korea, to overcome the burden of drug resistant TB, a sustainable and comprehensive TB control strategy that focuses on both DS-TB and MDR-TB is necessary.
\end{abstract}

TB continues to be a threat to public health. Only $39 \%$ of the estimated 484000 rifampinresistant (RR)/multidrug-resistant (MDR)-TB cases were reported, and worldwide the treatment success rate for RR/MDR-TB was $56 \%$ in the 2016 cohort. ${ }^{4}$ It is clear that to achieve the objectives of the END TB strategy, the expansion of TB control programmes, including the preventive treatment of persons at high risk, early diagnosis of TB and improvement of treatment outcomes is essential. 
Because of the slow disease dynamics of TB, mathematical and computational models are useful in forecasting the long-term evolution of the TB epidemic and for planning and resource allocation in public health. Therefore, several mathematical simulation models have been combined to predict the effect of public health interventions on TB control programmes. ${ }^{5-7}$ However, there are few models for the prediction of drug-resistant TB epidemiology that incorporate drug-susceptible (DS)TB. Drug-resistant TB is a significant challenge in the Korean peninsula. North Korea is one of the 30 countries with the highest TB and RR/MDR-TB burdens and the reported and estimated number of TB has increased (345/100000 in 2010 to 513/100000 in 2018). ${ }^{4}$ Among high-income countries, South Korea also has a considerable TB burden. The reported incidence was 96.4/100 000 in 2010 and 65.9/100 000 in 2018. ${ }^{8}$ Even though South Korea has achieved considerable reduction of TB in the past, TB incidence in South Korea is seven times higher than the average incidence for member countries of the Organization for Economic Co-Operation and Development. ${ }^{1}$

Recently, molecular test for raid diagnosis of TB and rifampin resistance are rolling out globally and several studies showed promising impact of this intervention for TB and RR/MDR-TB control. ${ }^{9}{ }^{10}$ Also, newly introducing new drugs for RR/MDR-TB treatment such as bedaquiline and delamanid suggest possible all oral regimens and improved outcome of RR/MDR-TB. ${ }^{11} 12$

The purpose of this study was to develop a mathematical model that accounts for recent trends in DS-TB and $\mathrm{RR} / \mathrm{MDR}-\mathrm{TB}$ incidence and to predict TB burden based on the proposed optimal TB control intervention strategy in South and North Korea.
We developed a deterministic compartmental model by incorporating the recent understanding of TB epidemiology, which aims to compare various intervention scenarios in response to TB. The model allows a systemic approach to planning control strategies considering multiple aspects of TB in South and North Korea.

\section{METHODS \\ Study design}

We constructed a deterministic compartmental model for TB. The basic structure of the TB model follows the Susceptible-Exposed-Infectious-Treated model but was modified by considering additional factors to reflect the characteristics of TB. We divided the latent TB infection compartment into fast progressors, E (approximately $5 \%$ of healthy adults will develop the active disease within 2 years), and slow progressors, L (individuals who do not have rapid progression). In addition, we differentiated between DS-TB and RR/MDR-TB populations to predict the long-term incidence of TB. Independent experiments were performed for South and North Korea. Our main interest is predicting the future incidence of $\mathrm{TB}$ and the number of TB deaths in South and North Korea by 2040 . We also performed a sensitivity analysis to assess the reliability of the predictions.

\section{Patient and public involvement}

Patients were not involved in this study.

\section{Mathematical model}

The model population was compartmentalised and classified by the status of the disease: susceptible (S), latent infection (E and L), infectious (I), treated (T), untreated (UT) and relapsed (R). The population was also divided

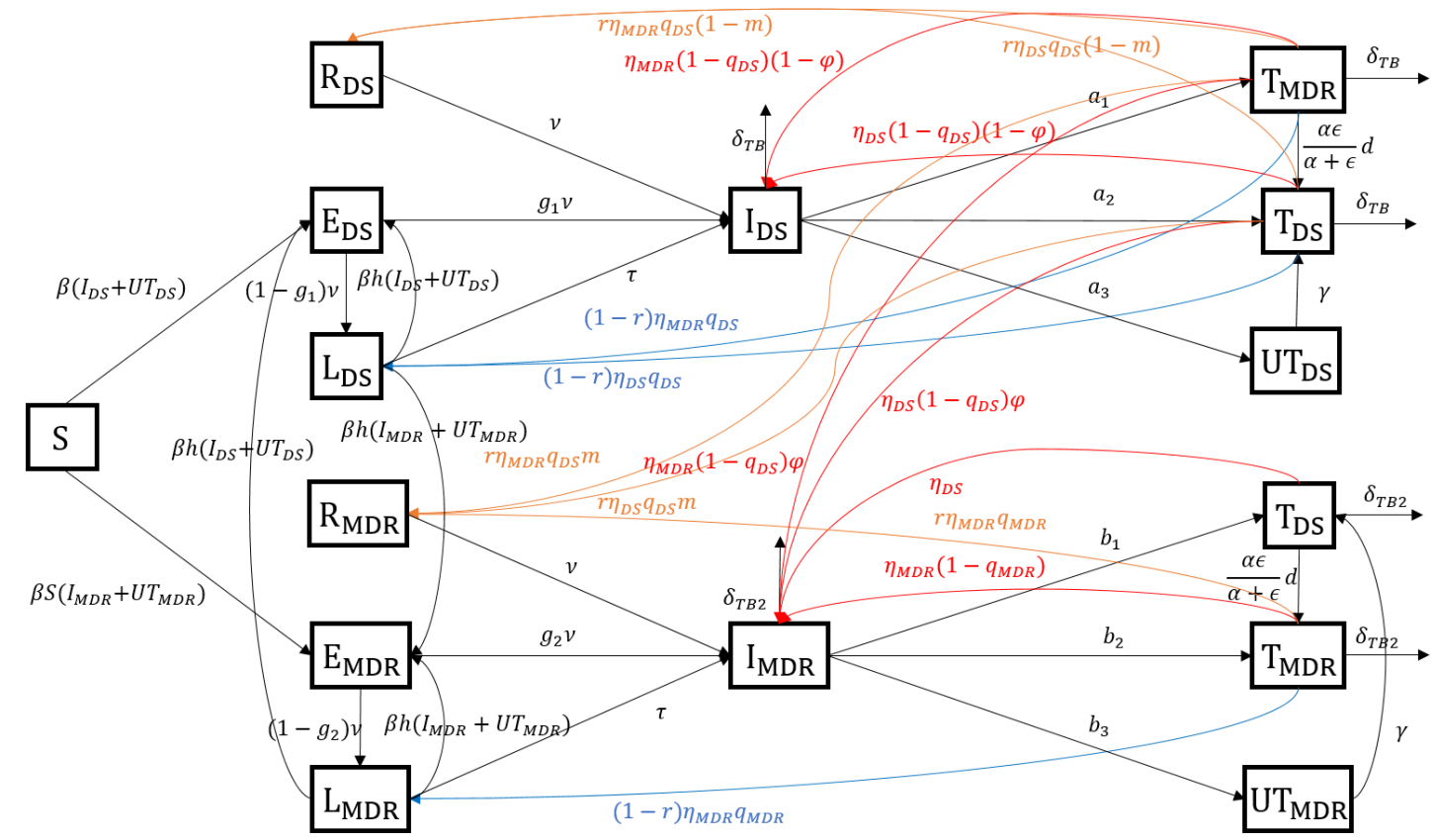

Figure 1 Diagram of TB transmission model. TB, tuberculosis. 


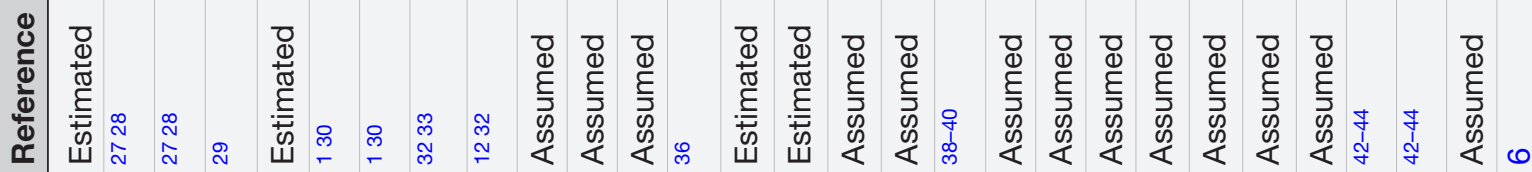

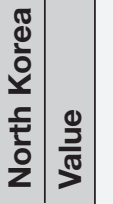

음

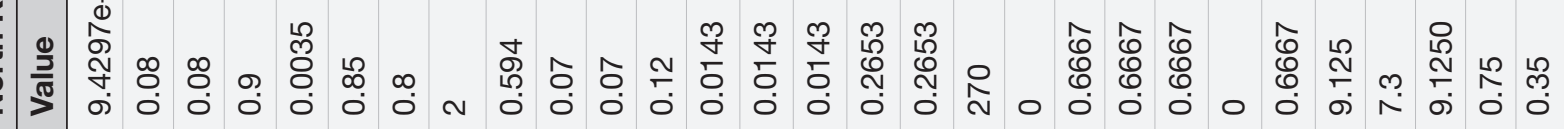

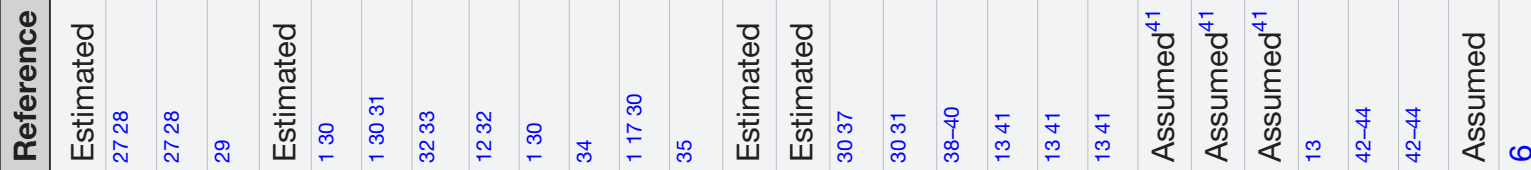

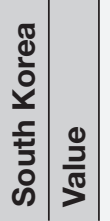

은

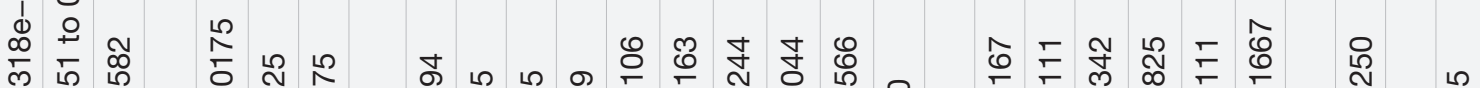
ம⿻

蒙

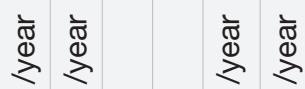

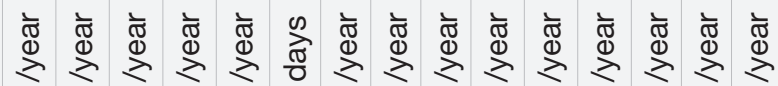

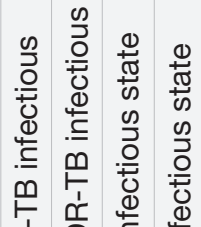

它

O

$\frac{\sqrt{0}}{\stackrel{\varpi}{0}}$

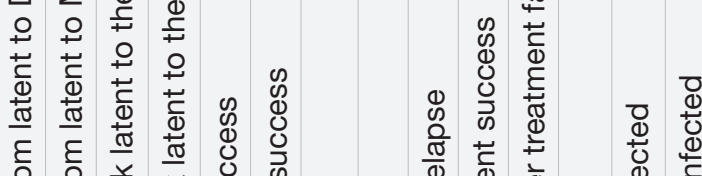

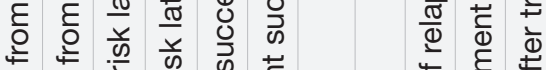

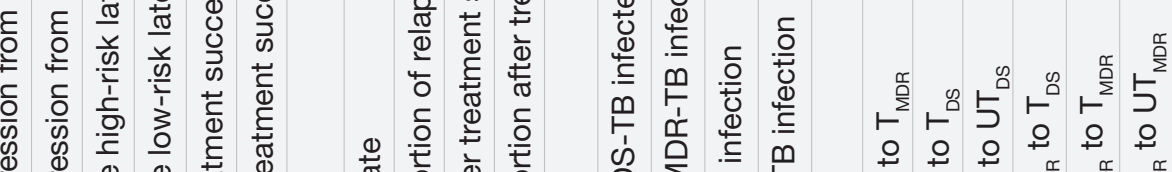

ब

它

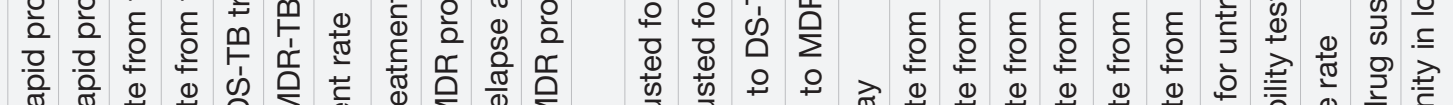

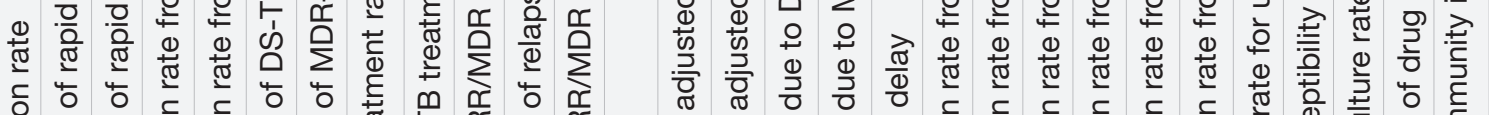


according to susceptibility to TB drugs (DS or RR/MDR). Figure 1 shows a flow diagram of a dynamic TB transmission model.

Briefly, the key model assumption includes all TB-infected individuals harbouring a single TB strain with a specified drug resistance pattern at any particular time. $\mathrm{RR} / \mathrm{MDR}-\mathrm{TB}$ is acquired by being infected with resistant strains, by de novo mutations during non-curative therapy, or relapse. Individuals who progress to the treatment $(\mathrm{T})$ stage can be placed in three categories. The basic case of transition from the infectious state to the treated state is $T_{D S}\left(a_{2}, b_{1}\right)$. When patients with RR/MDR-TB are started on the DS-TB treatment $\left(b_{1}\right)$, they can move to the $T_{M D R}$ state after drug susceptibility test results are obtained. In the second category, diagnosed patients progress to the $T_{M D R}$ state if RR/MDR-TB is immediately diagnosed using Xpert MTB/RIF $\left(b_{2}\right)$ or if DS-TB is misdiagnosed $\left(a_{1}\right)$. Patients with misdiagnosed DS-TB can move back to the $T_{D S}$ state with drug susceptibility test results. Progression to the untreated state (UT) occurs when the diagnosis is further delayed in the medical institutions by the AFB smear testing negative or TB-PCR $\left(a_{3}, b_{3}\right)$. Then, they progress to the $T_{D S}$ state at the rate of $\gamma$ after additional examination.

People in the slow progress latent (L) state are assumed to have partial immunity to $\mathrm{TB}$, which leads to a $35 \%$ reduction in the force of infection compared with people in the susceptible (S) state. ${ }^{6}$ Coinfection with HIV was not considered because such cases constitute a small proportion of all TB cases in South and North Korea.
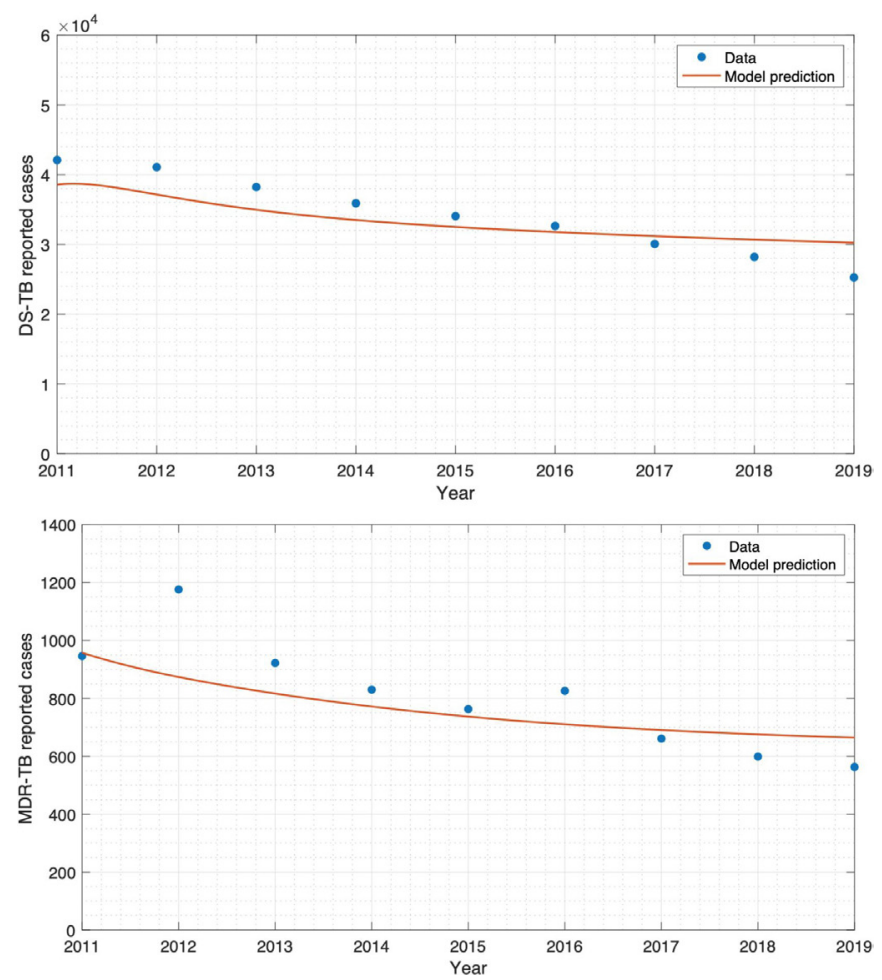

Figure 2 Model calibration results for South Korea. DS, drug-susceptible; MDR, multidrug-resistant; TB, tuberculosis.

\section{Data and parameters}

The parameter values were determined using published literature reviews, estimated, derived or assumed based on the best available local epidemiological data and mathematical formulas. Table 1 provides a summary of the descriptions and values used in the simulations.

In assessing the data of reported TB cases in South Korea, the number of reported cases is observed to have decreased rapidly since 2011. We hypothesised that this decline has been accelerated due to increased contact investigation and preventive treatment interventions compared with the previous period. Therefore, the proportion of rapid progression to active TB from the high-risk latent state has been deemed to decrease linearly between 2011 and 2019. Then, the transmission rate $\beta$ and progression rate from slow progress latent to infectious $\tau$ were estimated by fitting the model to the incidence data of TB in South Korea from 2011 to 2019 (figure 2). Maximum likelihood estimation technique was employed assuming Poisson distribution for the incidence data in model calibration.

For North Korea, the parameter values were determined similarly to those of South Korea, where global and regional WHO reports were used. The parameter values were adjusted using published literature reviews, values for South Korea and estimation based on the best available local epidemiological data.

\section{TB intervention strategy}

\section{South Korea}

The proportion of high-risk, latent to active TB decreased linearly from $5.1 \%$ to $4.4 \%$ from 2011 to 2019. Reflecting this trend, an additional level of preventive therapy was imposed until the proportion reached 3.6\% in 2025 for the baseline case (table 2).

Scenario 1-Preventive treatment control: Since preventive therapy affects the proportion of the high-risk latent to active TB group, we applied a gradual reduction of the flow from $\mathrm{E}$ to I to implement this intervention.

Scenario 2-Diagnostic improvement: In this scenario, the proportion of active TB would be improved with accurate diagnosis and proper treatment. This was adjusted by changing the proportion of the flow to the $T_{M D R}, T_{D S}$ and UT compartments. The baseline assumption was that $60 \%$ of DS-TB and $51 \%$ of MDR-TB moved to the $T_{D S}$ state, which increased up to $80 \%$ in this scenario.

Scenario 3-Active case-finding strategy: The infectious period of active TB would be reduced by scaling up the discovery of active cases. Therefore, the third intervention was imposed by reducing the value of the diagnostic delay parameter for the infectious individuals to receive treatment. The baseline was assumed to be 180 days, and the intervention scenario was tested by reducing patient delay to 150 days.

Scenario 4-Scaling up the treatment success rate for active TB: This intervention was experimented on by changing the treatment success rate values of DS-TB and RR/MDR-TB. In the baseline case, the treatment success 
Table 2 Cumulative number of reported TB cases, annual new infection and death predictions under the baseline scenario in South Korea

\begin{tabular}{|c|c|c|c|c|c|}
\hline & 2020 & 2025 & 2030 & 2035 & 2040 \\
\hline Cumulative DS report & 29767 & 171926 & 305421 & 432715 & 554258 \\
\hline Cumulative MDR report & 655 & 3835 & 6897 & 9885 & 12810 \\
\hline Yearly new infection & 96825 & 91372 & 88220 & 85548 & 82925 \\
\hline Death by TB & 4582 & 4248 & 4023 & 3842 & 3673 \\
\hline
\end{tabular}

rates for DS-TB and RR/MDR-TB were $92.5 \%$ and $87.5 \%$, respectively. In this scenario, the treatment success rate was increased to $97.5 \%$ for each DS-TB and RR/MDR-TB.

All interventions, except changes to the treatment success proportion, were applied simultaneously to both DS-TB and RR/MDR-TB.

\section{North Korea}

For North Korea, all four scenarios in the South Korean intervention were similarly applied, but the model parameters for the interventions were adjusted to the North Korean situation.

\section{Sensitivity analysis}

Sensitivity analyses were performed to examine the robustness of the model simulation results regarding the uncertainty of the parameter values. We perturbed each parameter by $5 \%$ of their values and compared the
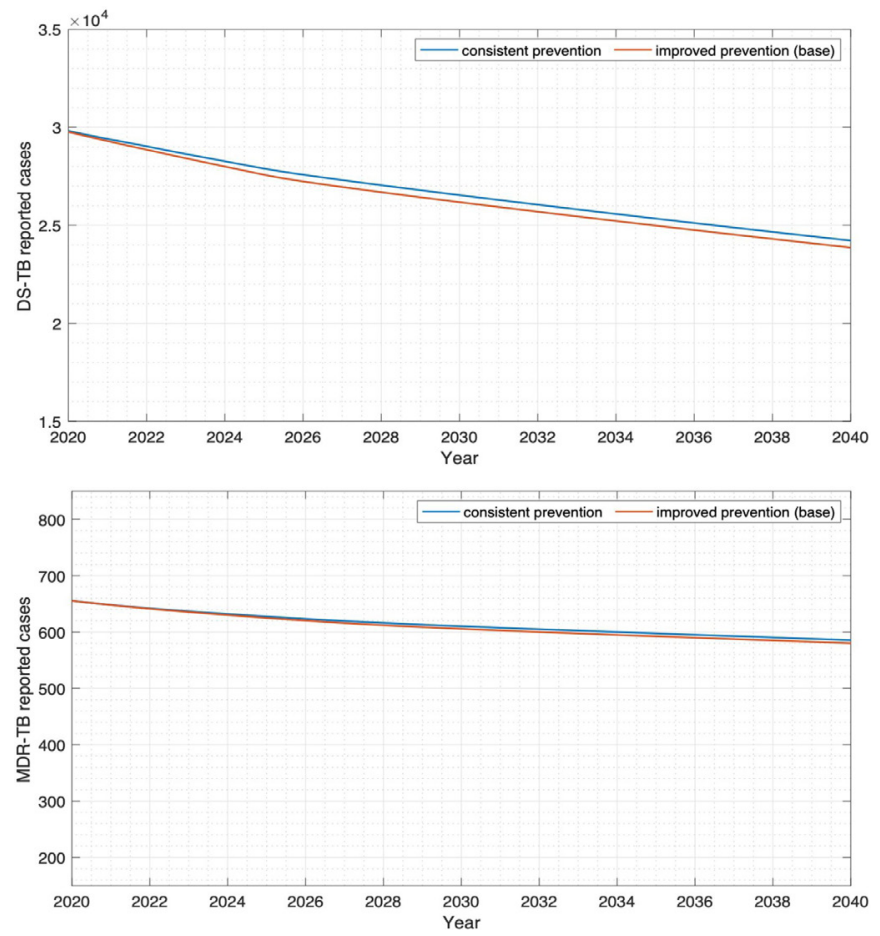

Figure 3 Number of reported TB cases with different preventive therapies implemented during 2020-2025 in South Korea. DS, drug-susceptible; MDR, multidrugresistant; TB, tuberculosis. number of DS-TB and RR/MDR-TB cases predicted in 2040.

\section{RESULTS \\ TB cases prevented by interventions in South Korea}

In the baseline case, the proportion of progression to active TB from the high risk latent group decreased linearly from $5.1 \%$ to $4.4 \%$ from 2011 to 2019 , and an additional level of preventive therapy was imposed until the proportion reached $3.6 \%$ by 2025 (table 2). Our model projected that incident cases of DS-TB and RR/MDR-TB, respectively, would drop to 27581 and 625 by 2025 . By maintaining a consistent setting after 2025, the number of new cases of DS-TB and RR/MDR-TB decreases slightly. The impact of four different TB control strategies on the incident cases was tested based on this situation.

To investigate the effects of preventive treatment intervention, the model was simulated assuming a consistent level of preventive therapy control from 2020 for the proportion to reach $3.9 \%$ in 2025 (table 2, figure 3). Without additional preventive therapy control from 2020 to 2025, the reported TB cases increase by 356 in 2040 and 6491 cumulatively from 2020 to 2040 (table 3, figure 3).

Regarding the diagnosis improvement intervention, $60 \%$ of patients with DS-TB were treated immediately without hospital delay and, in the baseline case, the remainder had an average hospital delay of 22 days. ${ }^{13}$ Only 9\% of patients with RR/MDR-TB started RR/MDR-TB treatment immediately, and 51\% initially received DS-TB treatment and started RR/MDR-TB treatment after the drug susceptibility test. In the diagnostic improvement control scenario, the proportion of patients without hospital delay was set at $80 \%$ for both DS-TB and RR/ MDR-TB (table 4, figure 4).

As a result, it was expected to reduce, respectively, the 3287 and 2380 additional DS-TB and RR/MDR-TB cases between 2020 and 2040. The impact of diagnostic improvement was more prominent in RR/MDR-TB cases.

The effect of the active case-finding strategy was estimated by reducing the time taken until the patient is treated. The baseline value was 180 days, which was shortened to 150 days in the intervention scenario (table 5, figure 5). 
Table 3 Cumulative number of reported TB cases, annual new infection and death predictions with consistent preventive therapy from 2020 to 2025 in South Korea

\begin{tabular}{lrrrrr}
\hline & \multicolumn{1}{c}{$\mathbf{2 0 2 0}$} & $\mathbf{2 0 2 5}$ & \multicolumn{1}{c}{$\mathbf{2 0 3 0}$} & \multicolumn{1}{c}{$\mathbf{2 0 3 5}$} & \multicolumn{1}{c}{$\mathbf{2 0 4 0}$} \\
\hline Cumulative DS report & 29818 & 173063 & 308331 & 437421 & 560749 \\
\hline Cumulative MDR report & 655 & 3842 & 6924 & 9937 & 12888 \\
\hline Yearly new infection & 96986 & 92391 & 89400 & 86735 & 84112 \\
\hline Death by TB & 4587 & 4290 & 4076 & 3896 & 3727 \\
\hline DS, drug-susceptible ; MDR, multidrug-resistant; TB, tuberculosis. & & &
\end{tabular}

Table 4 Cumulative number of reported TB cases, annual new infection and death predictions with diagnostic improvements in South Korea

\begin{tabular}{|c|c|c|c|c|c|}
\hline & 2020 & 2025 & 2030 & 2035 & 2040 \\
\hline Cumulative DS report & 29758 & 171354 & 304053 & 430437 & 550971 \\
\hline Cumulative MDR report & 622 & 3288 & 5756 & 8131 & 10430 \\
\hline Death by TB & 4563 & 4161 & 3931 & 3748 & 3577 \\
\hline
\end{tabular}

By reducing the delay to 150 days, the annual new infection rate significantly decreases to 64953 in 2040 , compared with 82925 in the baseline scenario. A cumulative reduction, respectively, of 20962 and 2584 reported DS-TB and RR/MDR-TB cases between 2020 and 2040 was expected.

Finally, the treatment success rate was improved by increasing the success rate for DS-TB from $92.5 \%$ to
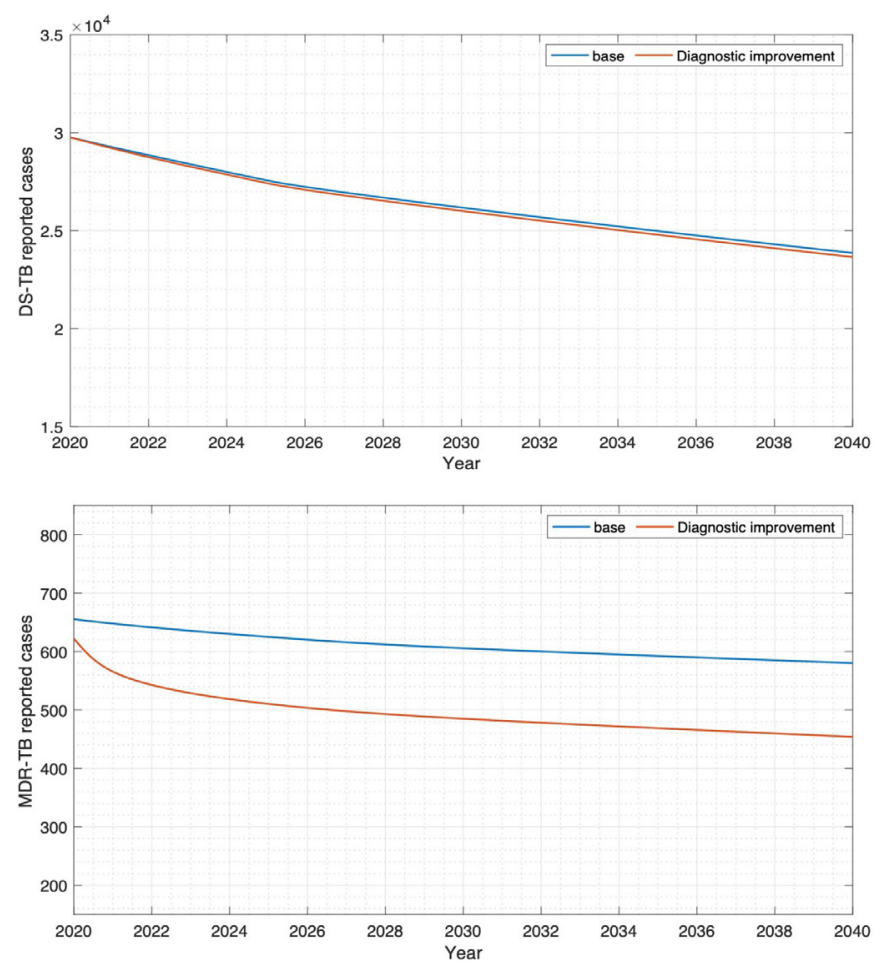

Figure 4 Number of reported TB cases with diagnostic improvement in South Korea. DS, drug-susceptible; MDR, multidrug-resistant; TB, tuberculosis.
$97.5 \%$ and the RR/MDR-TB success rate from $87.5 \%$ to $97.5 \%$ in a separate simulation (tables 6 and 7, figures 6 and 7). The improvement in the DS-TB treatment success rate, respectively, reduced the 25045 and 3876 cumulative reported cases of DS-TB and RR/MDR-TB between 2020 and 2040. Conversely, the control of RR/MDR-TB only decreased DS-TB cases by 4 and RR/MDR-TB cases by 1118 cumulatively during 2020 and 2040. It was observed that amelioration of the DS-TB treatment success rate had a greater impact on both the DS-TB and RR/MDR-TB cases.

\section{Sensitivity analysis in South Korea}

We measured the sensitivity of the TB burden in 2040 regarding the model parameters by perturbing $5 \%$ of the value of each parameter (figure 8 ). While the reported cases of DS-TB were most sensitive to the progression rate from the low-risk latent to infectious, MDR-TB was most sensitive to the proportion of DS treatment success. Diagnostic delay and transmission rate played key roles in both DS and MDR infection dynamics. The proportion of successful DS treatments, death rate adjusted for DS infection and the proportion of rapid progression from latent to DS-infectious were also significant in the results of the DS-TB cases. The MDR-TB cases were highly sensitive to the progression rate from the low-risk latent to infectious and the proportion of successful MDR treatments.

\section{Prevented TB cases by interventions and sensitivity analysis in North Korea}

The number of reported incident cases for DS-TB and estimated incident cases for RR/MDR-TB slightly increased from 2012 to 2015 and decreased from 2015 to 2019 in North Korea. All parameters were assumed to 
Table 5 Cumulative number of reported TB cases, annual new infection and death predictions with active case-finding in South Korea

\begin{tabular}{|c|c|c|c|c|c|}
\hline & 2020 & 2025 & 2030 & 2035 & 2040 \\
\hline Cumulative DS report & 31909 & 170422 & 298130 & 418910 & 533296 \\
\hline Cumulative MDR report & 696 & 3430 & 5810 & 8066 & 10226 \\
\hline Yearly new infection & 86530 & 73117 & 70073 & 67486 & 64953 \\
\hline Death by TB & 4308 & 3395 & 3174 & 3005 & 2847 \\
\hline
\end{tabular}

Table 6 Cumulative number of reported TB cases, annual new infection and death predictions with improvement of treatment success rate for DS-TB in South Korea

\begin{tabular}{lrrrrr}
\hline & \multicolumn{1}{c}{$\mathbf{2 0 2 0}$} & \multicolumn{1}{c}{$\mathbf{2 0 2 5}$} & \multicolumn{1}{c}{$\mathbf{2 0 3 0}$} & $\mathbf{2 0 3 5}$ & $\mathbf{2 0 4 0}$ \\
\hline Cumulative DS report & 29059 & 165060 & 292373 & 413612 & 529213 \\
\hline Cumulative MDR report & 582 & 2925 & 5014 & 7008 & 8934 \\
\hline Yearly new infection & 94356 & 86737 & 83625 & 80986 & 78394 \\
\hline Death by TB & 4514 & 4015 & 3790 & 3612 & 3446
\end{tabular}

DS, drug-susceptible ; MDR, multidrug-resistant; TB, tuberculosis.

Table 7 Cumulative number of reported TB cases, yearly new infection and death predictions with improvement of treatment success rate for MDR-TB in South Korea

\begin{tabular}{lrrrrr}
\hline & \multicolumn{1}{c}{$\mathbf{2 0 2 0}$} & \multicolumn{1}{c}{$\mathbf{2 0 2 5}$} & \multicolumn{1}{c}{$\mathbf{2 0 3 0}$} & \multicolumn{1}{c}{$\mathbf{2 0 3 5}$} & $\mathbf{2 0 4 0}$ \\
\hline Cumulative DS report & 29762 & 171915 & 305410 & 432707 & 554254 \\
\hline Cumulative MDR report & 632 & 3563 & 6349 & 9054 & 11692 \\
\hline Yearly new infection & 96735 & 91194 & 88040 & 85365 & 3827 \\
\hline Death by TB & 4579 & 4233 & 4008 & 3658
\end{tabular}

DS, drug-susceptible ; MDR, multidrug-resistant; TB, tuberculosis.

be constant from 2012 to 2019. Assuming all parameters remain constant, new cases of DS-TB decreased slightly, but cases of RR/MDR-TB were expected to increase gradually (online supplemental table S1).

The impact was assessed of four different TB control strategies on the incident cases, taking this situation as a baseline. The qualitative results are similar to South Korea in general. Please refer to online supplemental tables S1-1-S1-5 and figures S3-S6 for details.

We performed a sensitivity analysis of the TB burden in 2040 regarding model parameters in the same way as in South Korea (online supplemental figure S7). The results are almost identical in that the progression rate from the low-risk latent to infectious, the proportion of DS treatment success, diagnostic delay and transmission rate played significant roles in the DS-TB and MDR-TB infection dynamics.

\section{DISCUSSION}

New DS-TB and MDR-TB cases in Korea have steadily declined from 2011 to the present. According to the 2018 Tuberculosis Annual Report, ${ }^{8}$ the number of new patients with pulmonary TB decreased from 30100 new cases in 2011 to 20883 new cases in 2018. While the steady TB control strategy has reduced the TB incidence, based on the results of the simulation using a mathematical model, in the long term, the current decline is not expected to continue. By maintaining the current level of preventive control strategy until 2025, new TB cases are expected to decrease by only $14.5 \%$ from 2019 to 2040 , contrasted with the $19.9 \%$ reduction between 2011 and 2019. The long-term incubation period of TB is believed to be responsible for this mitigation. The latent TB infection rate among Koreans is $33.2 \%$, which is approximately 17 million people as of $2016 .{ }^{14}$ Our model simulations predicted that the number of people in the low-risk latent compartment would decrease very slowly. This is because none of the major TB control strategies directly target low-risk latent groups.

Contact investigation and preventive therapy for the close contact group had a significant impact on the reduction of new TB cases from 2011 to 2019. However, the long-term impact of preventive therapy for close contacts is not expected to be as effective as currently, due to TB having a long incubation period and a considerable population being latently infected in South Korea. 

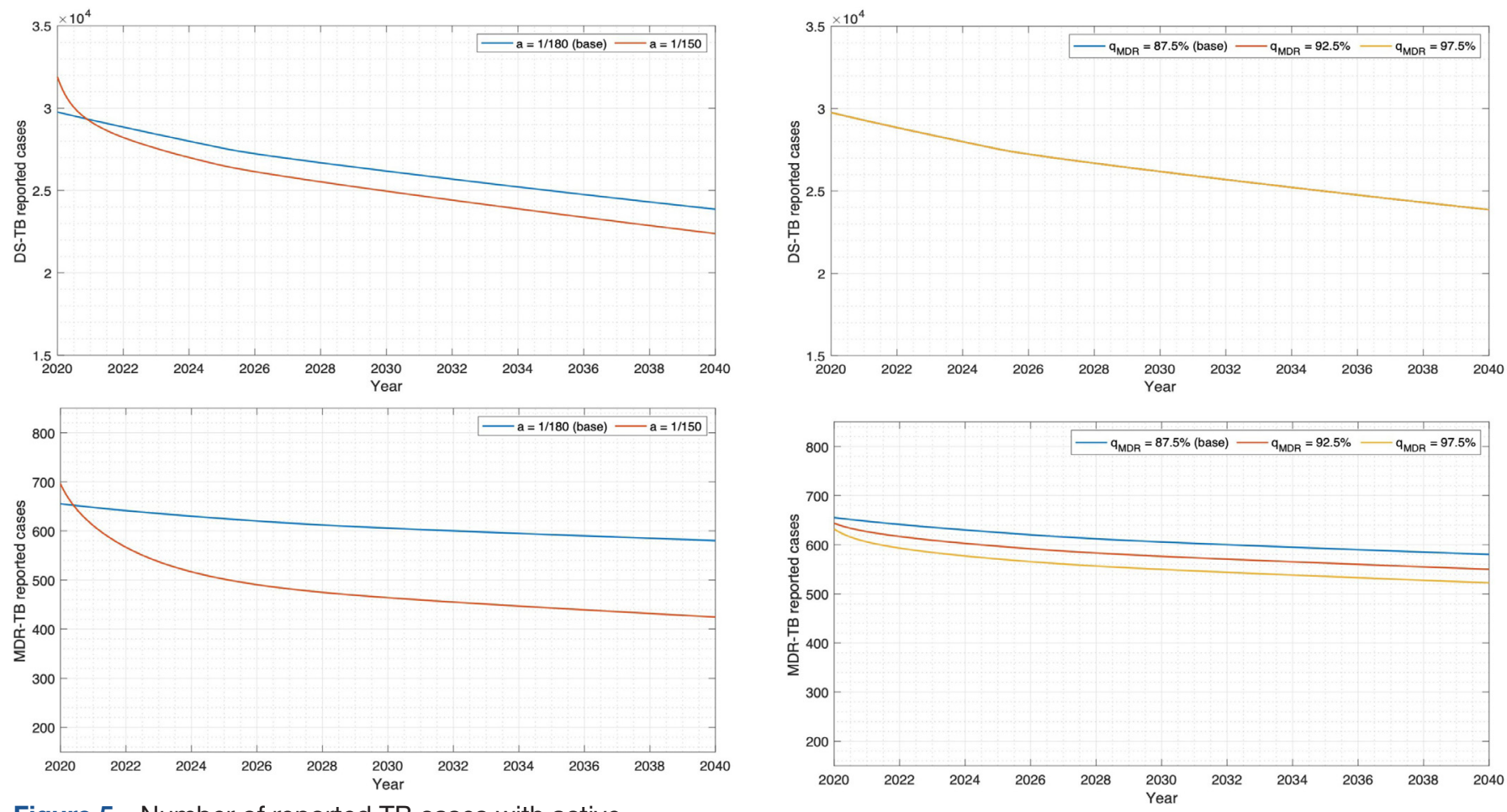

Figure 5 Number of reported TB cases with active case-finding in South Korea. DS, drug-susceptible; MDR, multidrug-resistant; TB, tuberculosis.
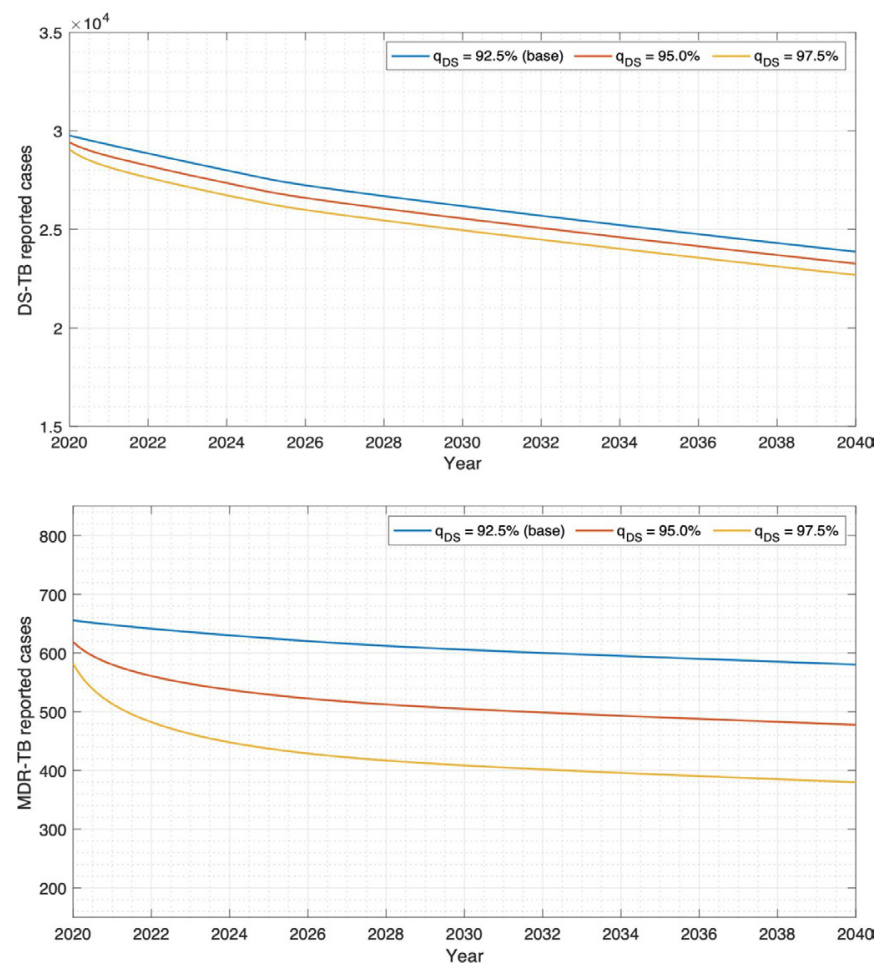

Figure 6 Number of reported TB cases with improvement of treatment success rate for DS-TB in South Korea. DS, drug-susceptible; MDR, multidrug-resistant; TB, tuberculosis.

Implementing the preventive therapy control from 2020 to $2025,1.5$ times the level of control during the period from 2011 to 2019, the decrease in new TB cases from 2019 to 2025 was only $8.5 \%$, while the reduction from

Figure 7 Number of reported TB cases with improvement of treatment success rate for RR/MDR-TB in South Korea. DS, drug-susceptible; MDR, multidrug-resistant; RR, rifampin-resistant; TB, tuberculosis.

2011 to 2019 was $19.9 \%$. Thus, a comprehensive TB control strategy is required to achieve the TB elimination goal in South Korea.

The quick and accurate diagnosis of TB and drug resistance was found to be more effective for RR/MDR-TB than for DS-TB. This is because, at the baseline, only
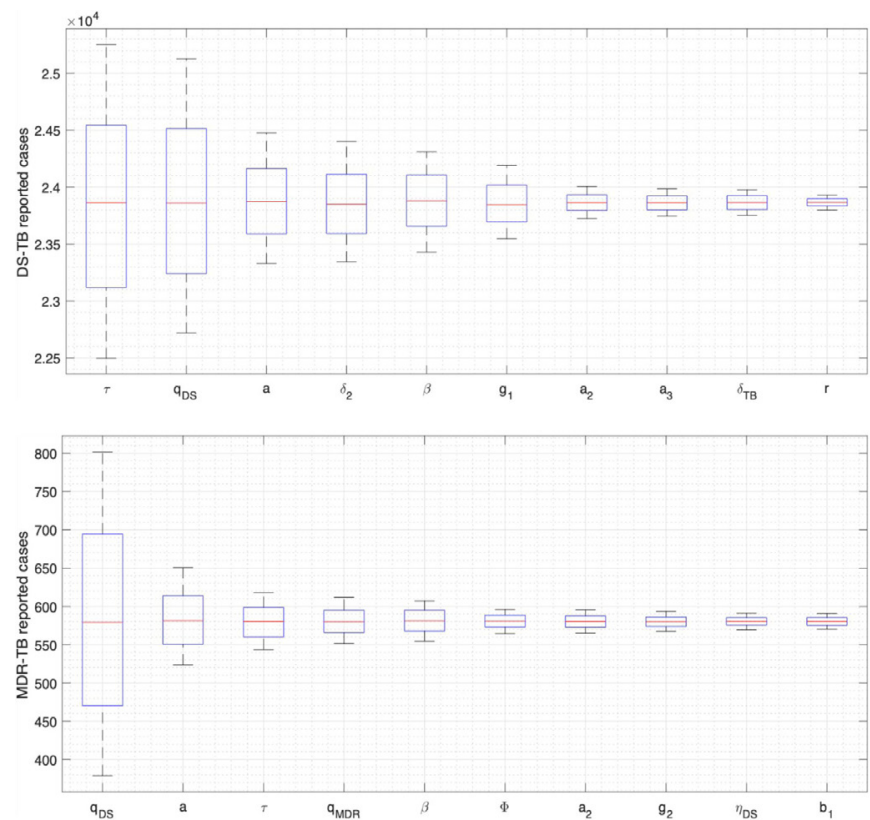

Figure 8 Sensitivity analysis of TB burden in 2040 regarding model parameters in South Korea. DS, drug-susceptible; MDR, multidrug-resistant; TB, tuberculosis. 


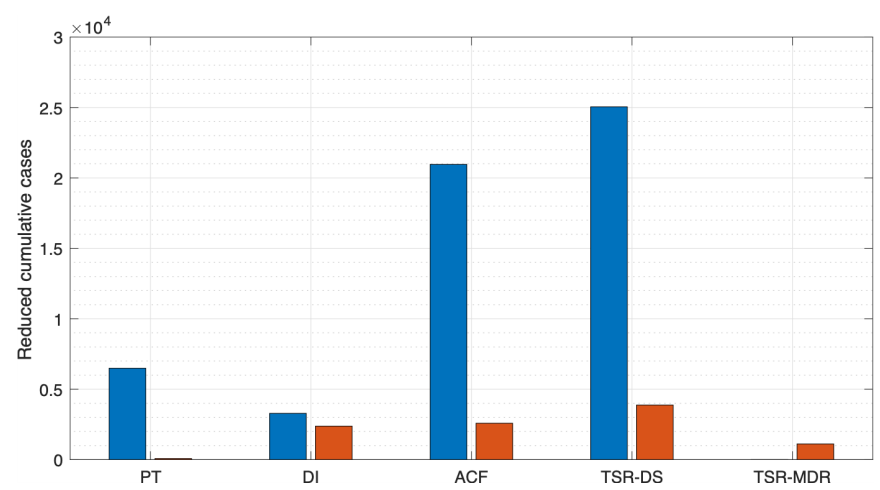

Figure 9 Reduction of cumulative TB cases from 20202040 under different intervention scenarios compared to the baseline in South Korea. Blue and red represent reductions in DS-TB and RR/MDR-TB cases, respectively. ACF, Active case-finding; DI, Diagnostic improvement; PT, Preventive treatment; TSR-DS, Treatment success rate for DS-TB; TSRMDR, Treatment success rate for RR/MDR-TB.

9\% of RR/MDR-TB cases are treated immediately without hospital delay; diagnosis of RR/MDR-TB can be improved more than for than DS-TB. This implies that the scale-up of rapid RR/MDR-TB diagnostic capacities such as rolling out of the Xpert MTB/RIF assay can help to reduce the number of cases of RR/MDR-TB in South Korea.

Worldwide, early case-finding and effective treatment are the principal strategies for controlling TB transmission and reduce incidence. ${ }^{1516}$ The improvement in the treatment success rate and the active case-finding strategy were found to have the most immediate effect among the interventions in our study (figure 9). The higher treatment success rate was substantially effective and meaningful in terms of RR/MDR-TB control in particular. The estimated proportion of TB cases with RR/MDR was $9.2 \%$ among previously treated cases in South Korea, which increased to $25 \%$ among re-treatment cases after treatment failure. ${ }^{47}$ If the treatment success rate for DS-TB increases, then the number of re-treatment patients decreases, which reduces the number of patients with RR/MDR-TB. The other policy is expected to decrease the number of low-risk latent groups in the long term by reducing the infectious period of $\mathrm{TB}$, which significantly curbs new infections.

Estimated data on reported TB cases in North Korea show that incidence rates remained constant between 2012 and $2019^{418}$ and preventive treatment strategy may be implemented in children under 5 years in familial contact based on WHO recommendation ${ }^{19}$ in North Korea. It might take time to implement active contact investigation and preventive treatment for all age in North Korea. Therefore, in the North Korean baseline scenario, preventive therapy control was assumed to remain constant from 2012 to 2040. Some parameters used in the North Korean model were determined in consideration of the resource limited situation in health system, malnutrition and high smoking rate compared with South Korea. Under these assumptions, it is predicted that the number of new TB cases will not decrease significantly.

In North Korea, the active case-finding was found to be most effective followed by preventive treatment. In our predicted model, RR/MDR-TB cases consistently would be a huge burden in North Korea. In fact, the diagnostic and treatment capacities for RR/MDR-TB in North Korea are scarce. The Ministry of Public Health of North Korea first began diagnosing and treating MDR-TB in 2008 with support from the Eugene Bell Foundation (EBF), a non-governmental organisation. ${ }^{20}$ However, the EBF programme did not cover all of North Korea. In 2010, the Global Fund to Fight AIDS, TB, and Malaria (GFATM) began a major project to strengthen TB control in North Korea, ${ }^{21}$ but a small portion of this international aid was allocated for the treatment of drug-resistant $\mathrm{TB} .{ }^{22}$ In addition, the tight UN sanctions due to the nuclear programme of North Korea have made it difficult to maintain the humanitarian assistance operations for the management of TB and MDR-TB in North Korea. ${ }^{23}$ In fact, GFATM closed its grant for North Korea in June 2018 and reached new agreement in $2020 .{ }^{24}$ North Korea is one of the high burden country of both TB and RR/ MDR-TB. ${ }^{4}$ Unstable drug supply and delayed diagnosis of TB and drug resistance would further increase the development of drug resistance in North Korea. Even in our model, which assumed the stable supply of TB drugs and diagnostics, RR/MDR-TB in North Korea increased in 2040. Thus, to overcome the burden of RR/MDR-TB in North Korea, a sustainable and comprehensive TB control strategy that focuses on both DS-TB and RR/ MDR-TB is necessary.

\section{Limitations}

Despite the strengths, this study has several limitations. First, age was not considered in the model to describe TB infection dynamics. Progression rate from latent state to active infectious state in TB and death rate may differ according to age. ${ }^{25}$ Second, it is assumed that infectivity remains constant during patient delay. However, the infectivity of TB can vary depending on the degree of disease progression and coughing and may not be constant during the patient delay period. Third, we hypothesised that decline of TB cases has been accelerated due to increased contact investigation and preventive treatment interventions to calibrate the model. However, the source actions of the current trend of decreasing number of new TB cases are unclear, since South Korea has carried out several interventions simultaneously to reduce the incidence of TB. Some other factors, including patient delay, hospital delay and low-risk latent to infectious, may have contributed to the reduction in $\mathrm{TB}$ cases during this period. In addition, there was an expansion of universal health coverage for TB treatment as social protection and strengthening the occupational health programme for TB. However, due to the lack of evidence for the impact of these factors on TB incidence, they were considered constant. Fourth, external aspects of the disease, such as changes in disease-related perceptions, 
were not considered. Research on TB awareness showed that the rate of willingness to screen for TB improved from $53.2 \%$ in 2013 to $62.7 \%$ in $2015 .{ }^{26}$ This suggests that apart from direct interventions, improved awareness can also decrease patient delay. Finally, the values of many parameters were assumed because of the lack of available information in North Korea.

\section{CONCLUSION}

The results show that intervention strategies in the order of improvement of treatment success rate for DS-TB, active case-finding, preventive therapy, reduced hospital delay and improvement of treatment success rate for RR/MDR-TB in South Korea are effective (figure 9). In particular, the treatment success rate increment for DS-TB has the most effect in reducing RR/MDR-TB. In North Korea, active case finding has the greatest impact on new TB incidence followed by the preventive therapy and treatment success rate for DS-TB (figure 8).

\section{Author affiliations}

${ }^{1}$ School of Mathematics and Computing, Yonsei University, Seodaemun-gu, South Korea

${ }^{2}$ Department of Internal Medicine, Yonsei University College of Medicine, Seodaemun-gu, South Korea

${ }^{3}$ Internal Medicine, Yonsei University College of Medicine, Seoul, South Korea

${ }^{4}$ Korean National Tuberculosis Association, Seoul, South Korea

${ }^{5}$ Institute of Immunology and Immunological Disease, Yonsei University College of Medicine, Seoul, South Korea

Contributors JSY, JYC, YAK and JL conceived the study and contributed to project administration. HC, JS and JL performed the primary modelling analysis. YP, HJK and YAK contributed to data collection. HC, YP and JS wrote the first draft of the report. JSY, JYC, YAK and JL contributed to the study design. All authors contributed to data interpretation and revision of the report.

Funding The present study was supported under the framework of the international cooperation program managed by the National Research Foundation of Korea (2019K1A5A2077463, FY2019) and by NRF-2015R1A5A1009350.

Disclaimer The funding source had no role in the study process, including the design, sample collection, analysis or interpretation of the results.

Competing interests None declared.

Patient consent for publication Not applicable.

Provenance and peer review Not commissioned; externally peer reviewed.

Data availability statement Data are available in a public, open access repository. The data source is included in the reference.

Supplemental material This content has been supplied by the author(s). It has not been vetted by BMJ Publishing Group Limited (BMJ) and may not have been peer-reviewed. Any opinions or recommendations discussed are solely those of the author(s) and are not endorsed by BMJ. BMJ disclaims all liability and responsibility arising from any reliance placed on the content. Where the content includes any translated material, BMJ does not warrant the accuracy and reliability of the translations (including but not limited to local regulations, clinical guidelines, terminology, drug names and drug dosages), and is not responsible for any error and/or omissions arising from translation and adaptation or otherwise.

Open access This is an open access article distributed in accordance with the Creative Commons Attribution Non Commercial (CC BY-NC 4.0) license, which permits others to distribute, remix, adapt, build upon this work non-commercially, and license their derivative works on different terms, provided the original work is properly cited, appropriate credit is given, any changes made indicated, and the use is non-commercial. See: http://creativecommons.org/licenses/by-nc/4.0/.

ORCID iD

Joon Sup Yeom http://orcid.org/0000-0001-8940-7170

\section{REFERENCES}

1 World Health Organization. Global tuberculosis report. Geneva: World Health Organization, 2020.

2 World Health Organization. The end TB strategy. Geneva: World Health Organization, 2015.

3 Sahu S, Ditiu L, Zumla A. After the UNGA high-level meeting on Tuberculosis-what next and how? Lancet Glob Health 2019;7:e558-60.

4 World Health Organization. Global tuberculosis report. Geneva: World Health Organization, 2019.

5 Dye C, Glaziou P, Floyd K, et al. Prospects for tuberculosis elimination. Annu Rev Public Health 2013;34:271-86.

6 Abu-Raddad LJ, Sabatelli L, Achterberg JT, et al. Epidemiological benefits of more-effective tuberculosis vaccines, drugs, and diagnostics. Proc Natl Acad Sci U S A 2009;106:13980-5.

7 Verguet S, Riumallo-Herl C, Gomez GB, et al. Catastrophic costs potentially averted by tuberculosis control in India and South Africa: a modelling study. Lancet Glob Health 2017;5:e1123-32.

8 Korea Centers for Disease Control and Prevention. Annual report on the notified tuberculosis in Korea, 2018.

9 Oxlade O, Falzon D, Menzies D. The impact and cost-effectiveness of strategies to detect drug-resistant tuberculosis. Eur Respir J 2012;39:626-34

10 Vassall A, van Kampen S, Sohn H, et al. Rapid diagnosis of tuberculosis with the Xpert MTB/RIF assay in high burden countries: a cost-effectiveness analysis. PLoS Med 2011;8:e1001120.

11 Schnippel K, Ndjeka N, Maartens G, et al. Effect of bedaquiline on mortality in South African patients with drug-resistant tuberculosis: a retrospective cohort study. Lancet Respir Med 2018;6:699-706.

12 World Health Organization,. WHO operational handbook on tuberculosis. Module 4: treatment - drug-resistant tuberculosis treatment, 2020.

13 Kwak SH, Choi JS, Lee EH, et al. Characteristics and risk factors associated with missed diagnosis in patients with smear-negative pulmonary tuberculosis. Korean J Intern Med 2021;36:S151-9.

14 Kyung Hyun Oh HJK, Oh SY, Lee JB, et al. 7th Korea National health and nutrition examination survey 1 st year(2016) tuberculin survey support, 2017.

15 Wingfield T, Verguet S. Active case finding in tuberculosis-affected households: time to scale up. Lancet Glob Health 2019;7:e296-8.

16 World Health Organization. Global strategy and targets for tuberculosis prevention care and control after, 2015.

17 Park Y-S, Hong S-J, Boo Y-K, et al. The National status of tuberculosis using nationwide medical records survey of patients with tuberculosis in Korea. Tuberc Respir Dis 2012;73:48.

18 Bhatia V. rGLC country support mission report: democratic peoples' Republic of Korea, 2019.

19 World Health Organization. WHO consolidated guidelines on tuberculosis. Module 1: prevention - tuberculosis preventive treatment, 2020.

20 Seung KJ, Franke M, Linton SW. Multidrug-resistant tuberculosis treatment in North Korea: is scale-up possible? PLoS Med 2016;13:e1002062.

21 Lee H, Ahn DY, Choi S, et al. The role of major donors in health aid to the democratic people's Republic of Korea. J Prev Med Public Health 2013;46:118-26.

22 Seung KJ, Linton SW. The growing problem of multidrug-resistant tuberculosis in North Korea. PLoS Med 2013;10:e1001486.

23 Burki T. North Korea and the global fund. Lancet Infect Dis 2018;18:501.

24 The Global Fund. Available: http://theglobalfund.org [Accessed 20 Aug 2021].

25 Comstock GW, Livesay VT, Woolpert SF. The prognosis of a positive tuberculin reaction in childhood and adolescence. Am J Epidemiol 1974:99:131-8.

26 Cho KS. Tuberculosis control in the Republic of Korea. Health Soc Welfare Rev 2017;37:179-212.

27 Cadena AM, Fortune SM, Flynn JL. Heterogeneity in tuberculosis. Nat Rev Immunol 2017;17:691-702.

28 Drain PK, Bajema KL, Dowdy D, et al. Incipient and subclinical tuberculosis: a clinical review of early stages and progression of infection. Clin Microbiol Rev 2018;31 doi:10.1128/CMR.00021-18

29 Sloot R, Schim van der Loeff MF, Kouw PM, et al. Risk of tuberculosis after recent exposure. A 10-year follow-up study of contacts in Amsterdam. Am J Respir Crit Care Med 2014;190:1044-52.

30 WHO Tuberculosis data. Available: https://www.who.int/teams/ global-tuberculosis-programme/data

31 Lee M, Han J, Kim YR, et al. Multidrug-resistant tuberculosis in South Korea: a retrospective analysis of national registry data in 2011-2015. Int J Tuberc Lung Dis 2019;23:850-7. 
32 Joint Committee for the Revision of Korean Guidelines for Tuberculosis. Korean guidelines for tuberculosis. 4th edn, 2020.

33 World Health Organization. Guidelines for treatment of drugsusceptible tuberculosis and patient care, 2017.

34 Lee H, Kim J. A study on the relapse rate of tuberculosis and related factors in Korea using nationwide tuberculosis notification data. Osong Public Health Res Perspect 2014;5:S8-17.

35 Korean Statistical Information Service,. Population trend survey, 2017.

36 United Nations. World population prospects. Volume 1 : Comprehensive tables 2015.

37 Kim Jinsun SJ, HyeKyung I, Eunhye S, Division of Tuberculosis Prevention and Control, Bureau of Infectious Disease Policy, Korea Disease Control and Prevention Agency (KDCA). Characteristics and trends in deaths from tuberculosis in Korea, 2020.

38 Sreeramareddy CT, Panduru KV, Menten J, et al. Time delays in diagnosis of pulmonary tuberculosis: a systematic review of literature. BMC Infect Dis 2009;9:91.
39 Getnet F, Demissie M, Assefa N, et al. Delay in diagnosis of pulmonary tuberculosis in low-and middle-income settings: systematic review and meta-analysis. BMC Pulm Med 2017;17:202

40 Vesga JF, Hallett TB, Reid MJA, et al. Assessing tuberculosis control priorities in high-burden settings: a modelling approach. Lancet Glob Health 2019;7:e585-95.

41 Horne DJ, Kohli M, Zifodya JS, et al. Xpert MTB/RIF and Xpert MTB/ RIF ultra for pulmonary tuberculosis and rifampicin resistance in adults. Cochrane Database Syst Rev 2019;6:CD009593.

42 The Korean society of clinical microbiology and National Institute of Health. Mannual of laboratory tests for tuberculosis 2013.

43 Clinical and Laboratory Standards Institute (CLSI). Laboratory detection and identification of mycobacteria. CLSI guideline M48. 2nd edn, 2018

44 Clinical and Laboratory Standards Institute (CLSI). Susceptibility testing of mycobacteria, nocardiae, and other aerobic actinomycetes. 3rd edn, 2018. 